\title{
Factors associated with pain and disability reduction following exercise interventions in chronic whiplash
}

Maria Landén Ludvigsson, Gunnel Peterson, A. Dedering, D. Falla and Anneli Peolsson

\section{Linköping University Post Print}

\section{Tweet}

N.B.: When citing this work, cite the original article.

Original Publication:

Maria Landén Ludvigsson, Gunnel Peterson, A. Dedering, D. Falla and Anneli Peolsson, Factors associated with pain and disability reduction following exercise interventions in chronic whiplash, 2016, European Journal of Pain, (20), 2, 307-315.

http://dx.doi.org/10.1002/ejp.729

Copyright: Wiley: 12 months

http://eu.wiley.com/WileyCDA/

Postprint available at: Linköping University Electronic Press

http://urn.kb.se/resolve?urn=urn:nbn:se:liu:diva-125302 


\title{
FACTORS ASSOCIATED WITH PAIN AND DISABILITY REDUCTION FOLLOWING EXERCISE INTERVENTIONS IN CHRONIC WHIPLASH.
}

\author{
Maria Landén Ludvigsson ${ }^{1,2}$, Gunnel Peterson ${ }^{1,3}$, Åsa Dedering ${ }^{4}$, Deborah Falla ${ }^{5,6}$, \\ Anneli Peolsson ${ }^{1}$ \\ ${ }^{1}$ Department of Medical and Health Sciences, Division of Physiotherapy, Linköping \\ University, Sweden \\ ${ }^{2}$ Rehab Väst, County Council of Östergötland, Sweden \\ ${ }^{3}$ Centre for Clinical Research Sörmland, Uppsala University, Sweden \\ ${ }^{4}$ Division of Physiotherapy, Department of Neurobiology, Care Sciences and Society, \\ Karolinska Institutet, and Department of Physical Therapy, Karolinska University Hospital, \\ Stockholm, Sweden \\ ${ }^{5}$ Pain Clinic, Center for Anesthesiology, Emergency and Intensive Care Medicine, University \\ Hospital Göttingen, Göttingen, Germany \\ ${ }^{6}$ Department of Neurorehabilitation Engineering, Bernstein Focus Neurotechnology (BFNT) \\ Göttingen, Bernstein Center for Computational Neuroscience, University Medical Center \\ Göttingen, Georg-August University, Göttingen, Germany
}

Category: Original article

Running head: Predicting exercise response in chronic whiplash

Conflicts of interest: The authors certify that they have no affiliations with or involvement in any organization or entity with any financial interest or non-financial interest in the subject matter or materials discussed in this manuscript.

Funding sources: The study was supported by funding from the Swedish government through the REHSAM foundation, and the regional Centers for Clinical Research of Östergötland and Sörmland County Councils, Medical Research Council of Southeast Sweden and Uppsala-Örebro Regional Research Council Sweden. AP was supported by the Swedish Research Council.

Correspondence : Maria Landén Ludvigsson, Linköping University, Department of Medical and Health Sciences, Division of Physiotherapy SE-581 83 Linköping, Sweden. Phone +46 10-103 1765, fax +46 1010317 06, e-mail: Maria.Landen.Ludvigsson@liu.se

\section{What's already known about this topic?}

- Exercise can be effective for people with chronic whiplash associated disorders (WAD) however the response is highly variable.

- Chronic WAD includes heterogeneous physical and psychological impairments. 


\section{What does this study add?}

- Factors associated with treatment response in chronic WAD differ in the short and long term, and depend on pain or disability as an outcome.

- Psychological factors were not associated with outcome.

- Neck-specific exercise increases the odds of pain/disability reduction compared to physical activity prescription. 


\section{ABSTRACT}

Background: Some studies support the prescription of exercise for people with whiplash associated disorders (WAD) however the response is highly variable. Further research is necessary to identify factors which predict response.

Methods: This is a secondary analysis of a randomized, multicenter controlled clinical trial of 202 volunteers with chronic WAD (grades 2 and 3). They received either neck-specific exercise with, or without a behavioral approach, or prescription of physical activity for 12weeks. Treatment response, defined as a clinical important reduction of pain or disability, was registered after 3 and 12 months, and factors associated with treatment response were explored using logistic regression.

Results: Participation in the neck-specific exercise group was the only significant factor associated with both neck pain and neck disability reduction both at 3 and 12 months. Patients in this group had up to 5.3 times higher odds of disability reduction, and 3.9 times higher odds of pain reduction compared to those in the physical activity group. Different baseline features were identified as predictors of response depending on the time point examined and the outcome measure selected (pain versus disability).

Conclusion: Factors associated with treatment response after exercise interventions differ in the short and long term and differ depending on whether neck pain or disability is considered as the primary outcome. Participation in a neck-specific exercise intervention, in contrast to general physical activity, was the only factor that consistently indicated higher odds of treatment success. These results support the prescription of neck-specific exercise for individuals with chronic WAD.

Key words: chronic, whiplash, prediction, exercise, pain, disability 


\section{Introduction}

Whiplash-associated disorders (WAD) are a significant public health problem with substantial social and economic costs (Jull et al., 2011) leaving up to 50\% of people with continuous neck pain one year after the injury (Carroll et al., 2008). Still there is no clear evidence of effective treatment (Verhagen et al., 2007). One explanation may be that those with persistent WAD form a heterogeneous group with variable and sometimes complex patterns of co-existing physical and psychological impairments (Sterling et al., 2003a; Sterling et al., 2003b). Thus, it is reasonable to expect that not all patients would benefit from the same intervention. This premise is supported by randomized controlled trials (RCT) on exercise for chronic WAD which have shown some variability of patient outcome (Stewart et al, 2007, Michaleff et al, 2014, Ludvigsson et al, 2015). Further research is warranted to identify factors which predict response.

In acute WAD a number of predictors of various outcomes have been presented but high initial neck pain appears to be the only factor which consistently predicts poor functional recovery (Ritchie et al., 2013). Predictive factors for symptom resolution may be different in a population with chronic pain however (Kamper et al., 2011). Stewart et al (Stewart et al., 2007) observed that higher baseline levels of pain and disability were associated with greater pain reduction, following exercise in a sample of patients with chronic WAD. Michaleff et al tested measures of central nervous hyperexcitability and psychological distress but failed to find any modifying effect for pain reduction following exercise (Michaleff et al., 2014). A recent study from our group on patients with chronic WAD showed a greater reduction in neck pain and neck-related disability after 3 and 6 months, following a neck-specific exercise program, with or without the addition of a behavioural approach, compared to the prescription of general physical activity (Ludvigsson, et al 2015). Although significant improvements were observed for the neck-specific training groups, there were both responders and nonresponders to treatment in all three groups. Interestingly, individuals classified as responders based on disability reduction were not always the same individuals classified as responders on pain reduction. However, factors associated with response were not evaluated. Indeed, there is an overall lack of clinical prediction rules that can be confidently applied to patients with WAD (Kamper et al., 2011) and to the best of our knowledge, there are no validated characteristics suggested to predict reduction of disability from exercise in patients with chronic WAD. 
The aim of this secondary analysis of a recent RCT (Ludvigsson, et al 2015) was to explore whether the type of exercise intervention (neck-specific exercise with or without a behavioral approach or prescription of physical activity) is a determinant of clinically important disability and/or pain reduction in individuals with chronic WAD, grades 2-3. In addition to evaluating the response to interventions in the short term we present longer term outcomes (12 months). Another aim was to assess whether features of the patients' baseline presentation are associated with these outcomes, following exercise interventions.

\section{Material and methods}

\subsection{Procedure and participants}

This is a secondary analysis, based on a randomized assessor blinded multicenter clinical trial with parallel design of three exercise interventions applied in people with chronic WAD (Ludvigsson, et al 2015) classified as grade 2 or 3 (Spitzer et al., 1995). Data was collected from February 2011to May 2013 and short term (3 and 6 month) outcomes have been previously presented (Ludvigsson, et al 2015). The current analysis also includes 12 month follow up data, not previously published. The study was approved by the Regional Ethics Committee of Linköping University, Sweden and the procedures were conducted according to the Declaration of Helsinki.

In accordance with the study protocol (Peolsson et al., 2013), participants were included if they were aged between 18-63 years, had experienced a whiplash injury in the preceding 6-36 months that was nominated as the cause of their current symptoms, had a Neck Disability Index (NDI) score (Vernon, 2008) of at least 10/50 points, and/or average pain on a Visual Analogue Scale (VAS) of $>20 / 100 \mathrm{~mm}(0=$ no pain, $100=$ worst imaginable pain) (Carlsson, 1983) for the preceding week. The exclusion criteria were signs of traumatic brain injury, previous neck trauma with unresolved symptoms, neck pain causing more than one month's work absence in the preceding year before the whiplash injury, myelopathy, spinal infection or tumor, previous neck surgery, more dominant pain elsewhere in the body, conditions potentially detrimental to completing the study interventions or insufficient competence of the Swedish language. Potential participants were identified and screened for eligibility by a four step process as previously described (Figure S1) (Ludvigsson, et al 2015). 
Randomization was performed by an independent researcher without any further involvement in the study and all data collection was performed by researchers blinded to allocation, as previously reported (Ludvigsson, et al 2015). Informed consent and baseline outcome measurements were collected before allocation. A total of 216 patients were included in the RCT (Ludvigsson, et al 2015). Out of these 216 patients, 14 were excluded from the analysis in the current study due to failure to attend at least one of the physiotherapy visits, rendering a total of 202 participants which constitutes this study sample. The 3 month follow-up after inclusion was completed by 190 patients (94\%), and 12 month follow-up was completed by 170 patients (84\%) (Figure S1). The mean age was 41 years (SD11), 64\% ( $n=129$ ) of the participants were female and $81 \%$ of them had experienced a motor vehicle accident as the cause of their WAD. Other causes included skiing or biking mishaps with acceleration/deceleration mechanism of energy transfer to the neck (Spitzer et al., 1995). Further baseline characteristics of the participants are presented in Table 1.

Table 1 Baseline characteristics and group allocation of participants with chronic whiplash-associated disorders ( $\mathrm{n}=202)$.

$\begin{array}{lc}\text { Intervention group, } \mathrm{n} & 201 \\ \text { NSE, } n(\%) & 70(35) \\ \text { NSEB, } n(\%) & 68(34)\end{array}$




$\begin{array}{lc}\text { PPA, } n \text { (\%) } & 63(31) \\ \text { Gender, female } \mathrm{n}(\%) & 129(64) \\ \text { Age, mean (SD) } & 41(11) \\ \text { Months since injury, mean (SD) } & 20(9) \\ \text { WAD grade 2, } \mathrm{n}(\%) & 115(57) \\ \text { WAD grade 3, } \mathrm{n}(\%) & 83(43) \\ \text { Sensibility disturbance, levels n (SD) } & 2(2) \\ \text { Disability, NDI, mean (SD) } & 17(7) \\ \text { Absence of dizziness, } \mathrm{n}(\%) & 46(23) \\ \text { Neck pain intensity (VAS), mean (SD) } & 42(24) \\ \text { Patient expectations (NRS), mean (SD) } & 6.9(2.2) \\ \text { Total ROM flex/ext, mean (SD) } & 111(27) \\ \text { Total ROM flexion, mean (SD) } & 44(15) \\ \text { Total ROM extension, mean (SD) } & 52(17) \\ \text { Total ROM rotation, mean, SD } & 96(26) \\ \text { Neck muscle end, flex, med (IQR) } & 21(10-44) \\ \text { Neck muscle end, ext, med (IQR) } & 46(18-118) \\ \text { Self-efficacy,SES, (mean) SD } & 150(36) \\ \text { Kinesophobia,TSK, mean (SD) } & 22(6) \\ \text { Pain catastrophizing,PCS, mean (SD) } & 18(9)\end{array}$

NSE, neck-specific exercise group; NSEB, neck-specific exercise group with a behavioural approach; PPA, prescription of physical activity group; WAD, whiplash-associated disorders, sensibility disturbance, numbers of cervical dermatomes (C4-C8) with sensibility disturbance; NDI, Neck Disability Index; VAS, Visual Analogue Scale; NRS, Numeric Rating Scale; Md, median; IQR, inter quartile range; Neck muscle end, flex = Neck muscle endurance flexors; NRS, Numeric Rating Scale; ROM, range of motion, Neck muscle end, Neck muscle endurance, presented as median due to highly skewed values; SES, Self-EfficacyScale; TSK, Tampa Scale for Kinesophobia (TSK-11) short form; PCS, Pain Catastrophizing Scale.

\subsection{Interventions}

The interventions were: 1. physiotherapist-led neck-specific exercise (NSE), 2. NSE with the addition of a behavioral approach (NSEB), or 3. prescription of physical activity (PPA). Exercise as a treatment in neck pain is considered to be safe, with temporary and benign side effects (Verhagen et al., 2007). All three interventions were undertaken over a 12 week period and were provided by physiotherapists in primary care. In the NSE-group participants undertook supervised neck-specific exercise twice weekly. Initially patients also practiced daily at home, focusing on gentle isometric neck movements aimed at facilitating activity of the deep cervical muscles. Gradually exercise was then introduced in the gym with progressive resistance training twice weekly with a focus on low load endurance training. A detailed description of the exercises can be found at the Academic Archive On-line (Landén Ludvigsson et al 2015). Exercise pain provocation was avoided in this group. Towards the end of the 12 week exercise period, participants were encouraged to continue neck-specific 
exercise at home and also engage in general physical activity. The protocol of exercises in the NSEB group was the same as that undertaken by the NSE group, but with the addition of a behavioural approach. In accordance with the concept of graded exercise, patients were encouraged not to focus on temporary increases in neck pain. They also received behavioral interventions including education and introduction to activities aimed at pain management and problem-solving, guided by a physiotherapist. Participants in the PPA group initially received a short motivational interview conducted by a physiotherapist, and based on the discussions within the interview and the subsequent physical examination, were prescribed individualized physical activity to be performed independently. Neck-specific exercises including any form of head resistance were not prescribed in this group. Further details about the intervention protocols have been published previously (Ludvigsson, et al 2015).

\subsection{Treatment response}

The outcomes at 3 and 12 months following commencement of the intervention were treatment response, defined as substantial pain reduction of $\geq 50 \%$ on a VAS scale (Dworkin et al., 2009), or a minimal clinical important difference (MCID) of neck related disability, defined as an NDI score reduction $\geq 5$ points out of 50 (Vernon, 2008). The NDI consists of 10 items grading neck-related disability from 0 (no activity limitations) to 5 (major activity limitations) with a total maximum score of 50 points (Vernon, 2008) with a higher score representing a higher level of disability. The validity and reliability of the NDI have been established (Vernon, 2008, MacDermid et al., 2009). The MCID of the NDI-score in neck pain populations has been tested versus global perceived change and a physician-rated change scale, and is suggested to be 3.5-5/50 points (Vernon, 2008). This also exceeds the measurement error in our study sample (minimal detectable difference 3.3 points) as previously reported (Ludvigsson, et al 2015). Current pain intensity was measured with a VAS-scale anchored by $0=$ no pain, and $100=$ worst imaginable pain (Carlsson, 1983). The Initiative on Methods, Measurement and Pain Assessment in Clinical Trials (IMMPACT) recommend reporting the proportion of patients achieving a certain degree of pain relief. A reduction in pain intensity of $\geq 50 \%$ is considered substantial improvement or treatment success (Dworkin et al., 2008). Completion rate of exercise during the interventions, collected from the physiotherapists and from participant exercise diaries was not significantly different between groups as previously reported (Ludvigsson, et al., 2015). Post-intervention recommended exercise adherence at one year was rated on a 4-point scale (full, fair, some or no adherence). Participant reporting some to full adherence were not significantly different 
between groups ( $p=0.23$, NSE, PPA; 79\%, NSEB; 69\%) even though there was a trend for lower attendance/adherence for the PPA at 3 months, and NSEB at one year.

\subsection{Potential predictive factors}

Baseline factors considered as potential predictors were selected based on a review of the literature and on clinical experience of two specialist physiotherapists with over 15 years’ experience managing patients following a whiplash injury (Kamper et al., 2010). Patients completed questionnaires at home, regarding their gender, age, dizziness (never or from time to time/more often), pain catastrophizing (the Pain Catastrophizing Scale, (PCS) 052, higher numbers indicating more pain catastrophizing (Osman et al., 2000)), kinesiophobia (Tampa Scale for Kinesiophobia, TSK-11 short form, score range 11-44, higher numbers indicating more fear of movement (Roelofs et al., 2007)), baseline neck pain intensity (VASscale 0-100), self-efficacy of performing various activities despite pain (Self Efficacy Scale, SES, 0-200, higher number indicating higher self-efficacy (Altmaier et al., 1993)), patient expectations of symptom relief from each of the three interventions (matched with each individual randomized intervention in the analysis) (NRS scale 0-10, $0=$ no expectations of help, 10 = best possible help), and disability (NDI, 0-50) (Vernon, 2008)). Clinical tests included neck flexor and extensor muscle endurance (time test recorded in seconds) (Peolsson et al., 2007), total active sagittal and rotation range of neck motion (degrees, measured with a CROM device (Capuano-Pucci et al., 1991)) and number of cervical levels of sensibility disturbance (brush or pinwheel, C4-C8 dermatomes). All data were collected at baseline prior to randomization.

\subsection{Statistical analysis}

The required sample size for the RCT was determined on the basis of the expected difference for the main outcome, NDI (3.5/50, SD 7), between any of the groups with an alpha level of $5 \%$ and a power of $80 \%$, allowing for $10 \%$ drop-outs, rendering a sample-size of 216. Patients were analyzed by original assigned groups. A sample-size of 10 subjects per variable in a regression equation is reported to be adequate (Beattie \& Nelson, 2006). Multivariable logistic regression models, using stepwise backward regression with $p \geq 0.1$ as a limit for removal of variables to reduce the risk of overlooking potential predictors were performed including all the variables as specified above. Group allocation was also included 
as a separate variable (Moons et al., 2009). Checks for multicollinearity were performed with the linear regression technique. A variance inflation factor (VIF) $>10$ or tolerance level $<0.1$ were used to denote significant multicollinearity (Field, 2009). Goodness of fit was assessed using the Hosmer-Lemeshow test (Field, 2009), and model assessment was made using Nagelkerke's pseudo $\mathrm{R}^{2}$. No variables were excluded due to multicollinearity (All VIF <3.1 and tolerance levels $>0.33$ ). Group comparisons of the significant variables found in the final step of the regression were performed using the Mann-Whiney U-test, independent sample ttest or chi-square as appropriate. The significance level was set at $\mathrm{p} \leq 0.05$. Analyses were performed with SPSS 22.0.

\section{Results}

\subsection{Treatment response}

At 3 months, a total of 77/202 patients (38\%) were classified as treatment responders when considering either neck pain or neck-related disability, but only 26 of them (34\% of the responders) were classified according to both. Twenty-four patients (31\% of the responders) were classified as treatment responders based on the reduction of disability only, and 27 (35\% of the responders) based on the reduction of pain only. At 12 months, a total of 79/202 patients (39\%) were classified as treatment responders with respect to pain or disability and 39 of them (49\% of the responders) were classified according to both. Twelve patients (15\% of the responders) were classified as treatment responders with respect to disability only, and 28 (36\% of the responders) with respect to pain only.

\subsection{Predictors of neck disability reduction}

The multivariable regression revealed that group allocation was a significant factor in relation to neck-related disability reduction, where patients in the neck-specific group had 4 times higher odds of achieving clinically important disability reduction after 3 months compared with the PPA group. There was no significant difference in odds between the NSEB group and the PPA group. The other significant factors were higher baseline NDI score, WAD grade 3, and higher neck extensor muscle endurance. At 12 months, group allocation was still a significant factor. Patients in the NSE group had 5.3 times, and in the NSEB group 6.4 times increased odds of achieving a reduction of neck disability compared to patients in the PPA group. The other significant factors associated with neck disability reduction were higher 
baseline NDI score, absence of dizziness and higher patient expectations (Table 2). There were no differences in baseline patient expectations between the three exercise groups ( $p=0.28$, Md NSE 7 (6-9), NSEB 7 (6-9), PPA 7 (5-8)). Neither gender, age, neck flexor muscle endurance nor any of the psychological questionnaires were predictive of disability reduction at either time point. Both models showed acceptable goodness of fit (HosmerLemeshow p>0.17).

Table 2. Factors associated with treatment response (reduced disability or pain) after 3 and 12 months in patients with chronic WAD after exercise interventions. Final step of the multivariable regression models.

Disability reduction (NDI) at 3 months $(n=184)$

Intervention group

B (SE) p-value Odds ratio (95\% CI)

NSE

NSEB

$1.39(0.53) \quad<\mathbf{0 . 0 1}$

4.02 (1.44-11.24)

$0.99(0.52) \quad 0.06 \quad 2.69(0.97-7.5)$ 


$\begin{array}{lccc}\text { NDI } & 0.07(0.03) & \mathbf{0 . 0 4} & 1.07(1.00-1.13) \\ \text { Sensibility disturbance, levels, } \mathrm{n} & -0,22(0.12) & 0.06 & 0.80(0.64-1.0) \\ \text { WAD grade 3 } & 0.98(0.45) & \mathbf{0 . 0 3} & 2.65(1.10-6.38) \\ \text { Neck muscle endurance, flex } & -0.02(0.01) & 0.08 & 0.99(0.97-1.0) \\ \text { Neck muscle endurance, ext } & 0.01(0.0) & <\mathbf{0 . 0 1} & 1.01(1.00-1.01) \\ \text { Constant } & -3.12(0.80) & 0.00 & 0.04\end{array}$

$\begin{array}{lccc}\begin{array}{l}\text { Disability reduction (NDI ) at } \mathbf{1 2} \text { months } \\ \text { (n=166) }\end{array} & \text { B (SE) } & \begin{array}{c}\text { p-value } \\ <\mathbf{0 . 0 1}\end{array} & \text { Odds ratio (95\% CI) } \\ & & & \\ \text { Intervention group } & 1.67(0.60) & <\mathbf{0 . 0 1} & 5.30(1.65-16.99) \\ \quad \text { NSE } & 1.86(0.60) & <\mathbf{0 . 0 1} & 6.41(2.00-20.62) \\ \text { NSEB } & 0.10(0.04) & <\mathbf{0 . 0 1} & 1.10(1.03-1.18) \\ \text { NDI } & -0.20(0.12) & 0.09 & 0.82(0.65-1.03) \\ \text { Sensibility disturbance, levels, n } & 1.50(0.56) & <\mathbf{0 . 0 1} & 4.49(1.49-13.53) \\ \text { Absence of dizziness } & 0.20(0.10) & \mathbf{0 . 0 5} & 1.23(1.00-1.50) \\ \text { Patient expectations } & & 0.08 & \\ \text { Activity level, IPAQ } & -1.23(0.68) & 0.07 & 0.29(0.08-1.10) \\ \text { Moderate } & 0.39(0.49) & 0.42 & 1.48(0.57-3.85) \\ \text { High } & -5.06(1.24) & 0.00 & 0.01 \\ \text { Constant } & & & \end{array}$

Pain reduction (VAS) at 3 months $(n=178)$

$\begin{array}{lccc} & \text { B (SE) } & \text { p-value } & \text { Odds ratio (95\% CI) } \\ \text { Intervention group } & & \mathbf{0 . 0 3} & \\ \text { NSE } & 1.35(0.52) & \mathbf{0 . 0 1} & 3.85(1.39-10.69) \\ \text { NSEB } & 1.10(0.53) & \mathbf{0 . 0 4} & 2.99(1.05-8.51) \\ \text { NDI } & -0.08(0.04) & \mathbf{0 . 0 4} & 0.92(0.85-1.0) \\ \text { Absence of dizziness } & 0.94(0.49) & 0.06 & 2.55(0.98-6.67) \\ \text { ROM rot } & 0.02(0.01) & \mathbf{0 . 0 1} & 1.02(1.00-1.04) \\ \text { Pain intensity (VAS) } & 0.03(0.01) & \mathbf{0 . 0 2} & 1.03(1.00-1.05) \\ \text { Constant } & -4.27(1.44) & <0.01 & 0.01\end{array}$

Pain reduction (VAS) at 12 months $(\mathrm{n}=162)$

\begin{tabular}{|c|c|c|c|}
\hline Intervention group & B (SE) & $\begin{array}{c}\text { p-value } \\
0.01\end{array}$ & Odds ratio $(95 \% \mathrm{CI})$ \\
\hline NSE & $1.31(0.46)$ & $<0.01$ & 3.70 (1.51-9.07) \\
\hline NSEB & $0.57(0.47)$ & 0.23 & $1.77(0.70-4.47)$ \\
\hline Absence of dizziness & $0.91(0.48)$ & 0.06 & $2.49(0.98-6.34)$ \\
\hline Constant & $-1.30(0.37)$ & 0.00 & 0.27 \\
\hline
\end{tabular}

NDI = Neck Disability Index, NSE = Neck-specific exercise group, NSEB $=$ Neck-specific exercise group with a behavioural approach, $\mathrm{WAD}=$ whiplash associated disorders, flex $=$ Neck flexors, ext $=$ Neck extensors, Sensibility disturbance, levels, $\mathrm{n}=$ number of cervical dermatomes with sensory disturbance, IPAQ = International Physical Activity Questionnaire, ROM rot = total range of motion in cervical rotation', Pain intensity $(\mathrm{VAS})=$ neck pain intensity (Visual Analogue Scale) .NDI reduction model Nagelkerke pseudo ${ }^{2}$ 
0.21 (at 3 months) and 0.31 (at 12 months). Hosmer and Lemeshow test goodness of fit $\mathrm{p}=0.80$ (at 3 months) and 0.17 (at 12 months)Pain reduction model Nagelkerke pseudo $R^{2} 0.24$ (at 3 months) and 0.13 (at 12 months). Hosmer and Lemeshow test goodness of fit $\mathrm{p}=0.11$ (at 3 months) and $\mathrm{p}=0.31$ (at 12 months).

\subsection{Predictors of pain reduction}

The multivariable regression analysis revealed that group allocation was a significant factor for neck pain reduction, where patients in both physiotherapist-led neckspecific exercise groups had higher odds of achieving substantial pain reduction after 3 months (NSE: 3.9, NSEB: 3.0 times higher odds compared to the PPA group). The other significant factors associated with pain reduction were higher baseline pain, larger range of motion (rotation) and lower NDI-score.

The only factor predictive of pain reduction after 12 months was group allocation. Patients in the NSE group had 3.7 times higher odds of achieving pain reduction compared to patients in the PPA group, but there was no difference in odds between the NSEB and PPA group (Table 2). Neither gender, age, patient expectations, neck muscle endurance nor any of the psychological scores were predictive of pain reduction at either time point. Both models showed acceptable goodness of fit (Hosmer-Lemeshow p>0.11) (Table 2).

\subsection{Group comparisons}

Higher baseline NDI was predictive of a clinically important reduction of neckrelated disability whereas lower NDI was predictive of neck pain reduction at three months. A comparison of baseline NDI scores between patients who were responders to pain and responders to disability only, confirmed that there was a significant difference $(\mathrm{p}<0.001)$ where the mean NDI score was 13 (SD 4) among those who were responders when classified by the extent of neck pain reduction, and 20 (SD 6) among those who were responders when classified by the extent of reduction of disability.

When comparing responders to treatment with non-responders with respect to neck pain reduction, there was a significant difference in group allocation at both time points where there were more responders in the two neck-specific exercise groups. Responders also had a larger baseline range of motion and lower NDI scores at 3 months. With respect to neckrelated disability, the only factor which was significant at either time point was group allocation with more responders in the two neck-specific exercise groups (Table S1). 
Table 3 ( S1 ) Table S1 Comparison between responders and non-responders to treatment regarding baseline factors significantly associated with treatment response (reduced disability or pain). Results after 3 and 12 months following exercise interventions in chronic whiplash associated disorders.

Disability reduction (NDI) at 3 months 


\section{Factor}

Intervention group

$\begin{array}{ll}\text { NSE, } n(\%)^{a} & 19(31) \\ \text { NSEB, } n(\%)^{a} & 24(37) \\ \text { PPA, } n(\%)^{a} & 9(16) \\ \text { NDI, mean (SD) } & 17.4(6.8) \\ \text { WAD grade 2/3, n (\%) } & 24 / 28(46 / 54) \\ \text { Neck muscle end, ext Md (IQR) } & 42(23-130)\end{array}$

Disability reduction (NDI ) at 12 months

Factor

Intervention group

$\begin{array}{ll}\text { NSE, } n(\%)^{a} & 20(35) \\ \text { NSEB, } n(\%)^{a} & 25(37) \\ \text { PPA, } n(\%)^{a} & 6(12) \\ \text { NDI, mean (SD) } & 17.4(6.8) \\ \text { Absence of dizziness, n (\%) } & 14(27) \\ \text { Patient expectations, Md (IQR) } & 8(6-8)\end{array}$

Pain reduction (VAS) at 3 months

treatment responders

$(n=53)$

\section{Factor}

Intervention group

$\begin{array}{ll}\text { NSE, } n(\%)^{a} & 24(39) \\ \text { NSEB, } n(\%)^{a} & 20(32) \\ P P A, n(\%)^{a} & 9(17) \\ \text { NDI, mean (SD) } & 14.7(5.07) \\ \text { ROM rot, mean (SD) } & 118(26) \\ \text { Pain intensity (VAS), mean (SD) } & 44(23)\end{array}$

Pain reduction (VAS) at 12 months

treatment responders $(n=67)$

Factor

Intervention group

$\begin{array}{ll}\text { NSE }, n(\%)^{a} & 29(52) \\ \text { NSEB, } n(\%)^{a} & 25(45) \\ \text { PPA, } n(\%)^{a} & 13(26)\end{array}$

non responders

$(n=132)$

p-value

0.03

43 (69)

$41(63)$

$48(84)$

$15.9(6.5)$

0.18

$80 / 52(61 / 39)$

0.07

$46(16-115)$

0.80

non responders

$(n=125)$

p-value

0.001

37 (65)

42 (63)

46 (82)

$15.9(6.5) \quad 0.18$

$22(20) \quad 0.27$

$7(5-8) \quad 0.08$

non responders

$(n=125)$

p-value

0.03

37 (61)

$43(68)$

45 (83)

17.7 (6.9)

$<0.01$

108 (28)

0.02

$44(24)$

0.92

non responders

p-value

$(\mathbf{n}=95)$

0.02

${ }^{\text {a }}$ denotes percentage within each intervention group

NSE $=$ Neck-specific exercise group, NSEB $=$ Neck-specific exercise group with a behavioural approach, WAD

$=$ whiplash associated disorders, $\mathrm{Md}=$ median, $\mathrm{IQR}=$ inter quartile range, ext $=$ Neck extensors, $\mathrm{ROM}$ rot $=$ 


\section{Discussion}

This study evaluated whether the type of exercise intervention is a determinant of clinically important neck disability or pain reduction in chronic WAD, and whether features of the patient's baseline presentation were associated with outcome following exercise interventions. The only significant factor associated with a reduction of both neck pain and neck-related disability at 3 and 12 months, was participation in the NSE group. Patients allocated to this group had up to 5.3 times higher odds of achieving disability reduction, and 3.9 times higher odds of achieving pain reduction compared to those in the PPA group. Participation in the NSEB group was associated with pain reduction at 3 months (odds ratio 3.0), and disability reduction at 12 months (odds ratio 6.4) compared to the PPA group but not for the other outcome/time point. The fact that exercise was supposed to be performed in a pain free manner in the NSE group may have contributed to the higher odds of pain reduction in this group. However the highest odds of disability reduction at 1 year was in the NSEB group (albeit not at 3 months), which may be due to the different approach encouraging patients to perform activities despite a temporary increase of their pain.

Comparisons between responders and non-responders to treatment confirmed that there were significant differences between intervention groups, where more responders belonged to the two physiotherapist-led neck-specific groups. There was no significant difference between groups regarding attendance/adherence, which could explain these results. Our results support the effect of neck-specific exercise interventions for patients with chronic WAD.

The mean duration of symptoms at baseline was almost 2 years in our study sample thus, it is unlikely that a significant improvement would have occurred without any intervention. Importantly, factors such as higher disability and WAD grade 3 are reported to be predictors of poor outcome (Walton et al., 2013) yet were associated with treatment response (neck disability reduction) in the current study. Patients with WAD grade 3 were 2.7 times more likely than those with WAD grade 2 to achieve disability reduction at three months, but the difference between responders and non-responders to treatment did not reach statistical significance. WAD grade was not associated with neck pain reduction. 
The result that higher baseline pain was a predictor of pain reduction is consistent with previous findings by Stewart et al (Stewart et al., 2007). However there was no significant difference between responders and non-responders in baseline pain intensity. The only factors apart from group allocation that were associated with pain reduction, and were also significantly different between responders to treatment and non-responders, were higher range of neck motion (rotation) and a lower NDI score. An interesting observation was that a higher NDI score was predictive of neck disability reduction, whereas a lower NDI score was predictive of neck pain reduction at three months. This illustrates the dilemma with the use of different outcomes and time points across different studies, and may partially explain the inconsistency of observed predictive factors between studies (Ritchie et al., 2013). Of all the patients classified as treatment responders, either regarding neck pain or disability, only one third were classified according to both outcomes at three months.

The absence of dizziness was a factor associated with reduction in neck disability, increasing the odds 4.5 times. The symptom of dizziness has been associated with a number of sensorimotor disturbances in people with chronic WAD including impaired balance, proprioception deficits and disturbances of oculomotor control (Treleaven et al., 2006; Treleaven et al., 2011). Thus very likely patients with dizziness require a specific intervention also targeting those features of sensorimotor control and not only neck-specific exercise which was employed in this study.

Other characteristics often suggested to be predictors of outcome in the acute phase, such as age and gender, were not associated with treatment outcome in this study sample of people with chronic WAD. Patient expectations has also been suggested to be predictive of outcome (Bishop et al., 2013), but was not predictive of the 3 month outcomes in the current study. Our result is consistent with previous findings in chronic WAD where treatment preference did not predict outcome (Stewart et al., 2008). However baseline patient expectations were associated with neck disability (but not pain) reduction at 12 months, even though the increase in odds was only 1.2 with each increasing number on the NRS (0-10). There were no differences in patient expectations between the three exercise groups which may explain this result. However, the difference between responders to treatment and nonresponders failed to reach significance.

Considering that exercise was the main focus in all three interventions it was surprising that baseline neck muscle endurance wasn't more strongly associated with 
outcome. However most patients had a baseline endurance time which was considerably lower than what has been documented in healthy populations (Peterson $\mathrm{G}$ et al, submitted 2014) indicating that there was the potential for substantial improvement for most patients. Moreover, specific measures of neuromuscular control of the neck, previously shown to be a feature of chronic neck pain (Falla et al., 2004) were not measured in this study.

Psychological factors were not associated with either outcome, which is consistent with a recent observation that the degree of psychological distress did not modify the effect of treatment in chronic WAD (Michaleff et al., 2014). Although speculative, this may be different in a population with greater baseline psychological distress, which may be more common in patients recruited from pain clinics (Mailis-Gagnon et al., 2007) than from primary care. Even though an analysis of individuals who declined to participate in this study showed that the study sample was well representative in age, gender and level of pain (Ludvigsson, et al., 2015) we have no measure of kinesophobia or pain catastrophizing in the individuals who declined participation. However the TSK and PCS scores in this study sample were similar to previously presented scores in other chronic pain conditions (Roefels et al 2007, Kjoegs et al 2014)

\section{Clinical implications}

The results of this study support the prescription of specific exercise for individuals with chronic WAD. Furthermore, the results provide an initial indication of the factors which can be important determinants for pain and disability reduction following specific exercise interventions. Predictors however are not a replacement for clinical judgment and should complement rather than replace clinical opinion and intuition (Beattie \& Nelson, 2006).

\section{Strengths and Limitations}

A strength of this study is that it includes data from one of the larger randomized controlled trials in the field. It was assessor blinded, and conducted at multiple primary care centers. Both self-rated and clinical measurements were collected. Individuals with WAD grade 3, often excluded in other studies, were included in this study, which adds more valuable knowledge about this group. However, there are also limitations of this study which 
should be acknowledged. Only exercise interventions were evaluated in the current study, thus it is unknown whether the features identified would predict response to other interventions. The only factor associated with both treatment outcomes at both time points was participation in a neck-specific exercise group, which indicates that it may be a more important mediating factor than any of the baseline characteristics and scores tested in this study, even after 12 months. Characteristics like educational, compensational, marital and work status were not included in the regression model, but were tested with a univariate regression without any significant association $(\mathrm{p}>0.39)$. However it is acknowledged that there may be other factors, not measured in this study, associated with treatment outcome. To maximize the clinical applicability of this work, only outcome measures which are possible for clinicians to implement in everyday practice were included.

Considering that the median per-claimant number of physiotherapy visits after a whiplash injury has been reported to be quite high (14 visits) (Grimmer-Somers et al., 2012), and the proposed number of visits in the most effective interventions in our study was even higher, it is important for future studies to identify predictors of who will benefit from specific treatments, and to evaluate the cost-effectiveness of that intervention.

\section{Conclusion}

Factors associated with treatment response after exercise interventions differ in the short and long term and differ depending on whether neck pain or disability is considered as the primary outcome. Participation in a neck-specific exercise intervention, in contrast to general physical activity, was the only factor that consistently indicated higher odds of treatment success. These results support the prescription of neck-specific exercise for individuals with chronic WAD.

\section{Acknowledgement}

We wish to thank all participants in this study, including WAD participants, physiotherapists and staff involved at any stage of the study.

\section{Author contributions}


Conception and design: MLL, GP, ÅD, AP, acquisition of data MLL, GP, analysis and interpretation of data MLL, ÅD, DF, AP, drafting of article MLL, critical revision MLL, GP, $\AA ̊ \mathrm{D}, \mathrm{DF}, \mathrm{AP}$, final approval MLL, GP, ÅD, DF, AP.

All authors have discussed the results and commented on the manuscript.

Clinical Study Registration Number NCT01528579

\section{REFERENCES}

Altmaier, E.M., Russell, D.W., Kao, C.F., Lehmann, T.R. \& Weinstein, J.N. (1993) Role of self-efficacy in rehabilitation outcome among chronic low back pain patients. Journal of Counseling Psychology, 40, 335-339. 
Beattie, P. \& Nelson, R. (2006) Clinical prediction rules: what are they and what do they tell us? Aust J Physiother, 52, 157-163.

Bishop, M.D., Mintken, P.E., Bialosky, J.E. \& Cleland, J.A. (2013) Patient expectations of benefit from interventions for neck pain and resulting influence on outcomes. J Orthop Sports Phys Ther, 43, 457-465.

Capuano-Pucci, D., Rheault, W., Aukai, J., Bracke, M., Day, R. \& Pastrick, M. (1991) Intratester and intertester reliability of the cervical range of motion device. Arch Phys Med Rehabil, 72, 338340.

Carlsson, A.M. (1983) Assessment of chronic pain. I. Aspects of the reliability and validity of the visual analogue scale. Pain, 16, 87-101.

Carroll, L.J., Holm, L.W., Hogg-Johnson, S., Cote, P., Cassidy, J.D., Haldeman, S., Nordin, M., Hurwitz, E.L., Carragee, E.J., van der Velde, G., Peloso, P.M. \& Guzman, J. (2008) Course and prognostic factors for neck pain in whiplash-associated disorders (WAD): results of the Bone and Joint Decade 2000-2010 Task Force on Neck Pain and Its Associated Disorders. Spine (Phila Pa 1976), 33, S83-92.

Dworkin, R.H., Turk, D.C., McDermott, M.P., Peirce-Sandner, S., Burke, L.B., Cowan, P., Farrar, J.T., Hertz, S., Raja, S.N., Rappaport, B.A., Rauschkolb, C. \& Sampaio, C. (2009) Interpreting the clinical importance of group differences in chronic pain clinical trials: IMMPACT recommendations. Pain, 146, 238-244.

Dworkin, R.H., Turk, D.C., Wyrwich, K.W., Beaton, D., Cleeland, C.S., Farrar, J.T., Haythornthwaite, J.A., Jensen, M.P., Kerns, R.D., Ader, D.N., Brandenburg, N., Burke, L.B., Cella, D., Chandler, J., Cowan, P., Dimitrova, R., Dionne, R., Hertz, S., Jadad, A.R., Katz, N.P., Kehlet, H., Kramer, L.D., Manning, D.C., McCormick, C., McDermott, M.P., McQuay, H.J., Patel, S., Porter, L., Quessy, S., Rappaport, B.A., Rauschkolb, C., Revicki, D.A., Rothman, M., Schmader, K.E., Stacey, B.R., Stauffer, J.W., von Stein, T., White, R.E., Witter, J. \& Zavisic, S. (2008) Interpreting the clinical importance of treatment outcomes in chronic pain clinical trials: IMMPACT recommendations. J Pain, 9, 105-121.

Falla, D., Bilenkij, G. \& Jull, G. (2004) Patients with chronic neck pain demonstrate altered patterns of muscle activation during performance of a functional upper limb task. Spine (Phila Pa 1976), 29, 1436-1440.

Field, A. (2009) Discovering statistics using SPSS. Sage Publications Ltd, London.

Grimmer-Somers, K., Milanese, S., Kumar, S., Brennan, C. \& Mifsud, I. (2012) Number and frequency of physiotherapy services for motor vehicle-induced whiplash: interrogating motor accident insurance data 2006-2009. Journal of rehabilitation medicine : official journal of the UEMS European Board of Physical and Rehabilitation Medicine, 44, 774-780. 
Jull, G.A., Sterling, M., Curatolo, M., Carroll, L. \& Hodges, P. (2011) Toward lessening the rate of transition of acute whiplash to a chronic disorder. Spine (Phila Pa 1976), 36, S173-174.

Kamper, S.J., Hancock, M.J. \& Maher, C.G. (2011) Optimal designs for prediction studies of whiplash. Spine (Phila Pa 1976), 36, S268-274.

Kamper, S.J., Maher, C.G., Hancock, M.J., Koes, B.W., Croft, P.R. \& Hay, E. (2010) Treatment-based subgroups of low back pain: a guide to appraisal of research studies and a summary of current evidence. Best Pract Res Clin Rheumatol, 24, 181-191.

Ludvigsson, M.L., Peterson, G., O'Leary, S., Dedering, A. \& Peolsson, A. (2015) The Effect of Neckspecific Exercise With, or Without a Behavioral Approach, on Pain, Disability, and Self-Efficacy in Chronic Whiplash-associated Disorders: A Randomized Clinical Trial. Clin J Pain, 31, 294303.

MacDermid, J.C., Walton, D.M., Avery, S., Blanchard, A., Etruw, E., McAlpine, C. \& Goldsmith, C.H. (2009) Measurement properties of the neck disability index: a systematic review. J Orthop Sports Phys Ther, 39, 400-417.

Mailis-Gagnon, A., Yegneswaran, B., Lakha, S.F., Nicholson, K., Steiman, A.J., Ng, D., Papagapiou, M., Umana, M., Cohodarevic, T. \& Zurowski, M. (2007) Pain characteristics and demographics of patients attending a university-affiliated pain clinic in Toronto, Ontario. Pain Res Manag, 12, 93-99.

Michaleff, Z.A., Maher, C.G., Lin, C.W., Rebbeck, T., Jull, G., Latimer, J., Connelly, L. \& Sterling, M. (2014) Comprehensive physiotherapy exercise programme or advice for chronic whiplash (PROMISE): a pragmatic randomised controlled trial. Lancet.

Moons, K.G., Royston, P., Vergouwe, Y., Grobbee, D.E. \& Altman, D.G. (2009) Prognosis and prognostic research: what, why, and how? BMJ, 338, b375.

Osman, A., Barrios, F.X., Gutierrez, P.M., Kopper, B.A., Merrifield, T. \& Grittmann, L. (2000) The Pain Catastrophizing Scale: further psychometric evaluation with adult samples. J Behav Med, 23, 351-365.

Peolsson, A., Almkvist, C., Dahlberg, C., Lindqvist, S. \& Pettersson, S. (2007) Age- and sex-specific reference values of a test of neck muscle endurance. J Manipulative Physiol Ther, 30, 171177.

Peolsson, A., Ludvigsson, M.L., Overmeer, T., Dedering, A., Bernfort, L., Johansson, G., Kammerlind, A.S. \& Peterson, G. (2013) Effects of neck-specific exercise with or without a behavioural approach in addition to prescribed physical activity for individuals with chronic whiplashassociated disorders: a prospective randomised study. Bmc Musculoskel Dis, 14. 
Ritchie, C., Hendrikz, J., Kenardy, J. \& Sterling, M. (2013) Derivation of a clinical prediction rule to identify both chronic moderate/severe disability and full recovery following whiplash injury. Pain, 154, 2198-2206.

Roelofs, J., Sluiter, J.K., Frings-Dresen, M.H., Goossens, M., Thibault, P., Boersma, K. \& Vlaeyen, J.W. (2007) Fear of movement and (re)injury in chronic musculoskeletal pain: Evidence for an invariant two-factor model of the Tampa Scale for Kinesiophobia across pain diagnoses and Dutch, Swedish, and Canadian samples. Pain, 131, 181-190.

Spitzer, W.O., Skovron, M.L., Salmi, L.R., Cassidy, J.D., Duranceau, J., Suissa, S. \& Zeiss, E. (1995) Scientific monograph of the Quebec Task Force on Whiplash-Associated Disorders: redefining "whiplash" and its management. Spine (Phila Pa 1976), 20, 1S-73S.

Sterling, M., Jull, G., Vicenzino, B., Kenardy, J. \& Darnell, R. (2003a) Development of motor system dysfunction following whiplash injury. Pain, 103, 65-73.

Sterling, M., Kenardy, J., Jull, G. \& Vicenzino, B. (2003b) The development of psychological changes following whiplash injury. Pain, 106, 481-489.

Stewart, M.J., Maher, C.G., Refshauge, K.M., Herbert, R.D., Bogduk, N. \& Nicholas, M. (2007) Randomized controlled trial of exercise for chronic whiplash-associated disorders. Pain, 128, 59-68.

Stewart, M.J., Maher, C.G., Refshauge, K.M., Herbert, R.D. \& Nicholas, M.K. (2008) Patient and clinician treatment preferences do not moderate the effect of exercise treatment in chronic whiplash-associated disorders. Eur J Pain, 12, 879-885.

Treleaven, J., Jull, G. \& Grip, H. (2011) Head eye co-ordination and gaze stability in subjects with persistent whiplash associated disorders. Man Ther, 16, 252-257.

Treleaven, J., Jull, G. \& LowChoy, N. (2006) The relationship of cervical joint position error to balance and eye movement disturbances in persistent whiplash. Man Ther, 11, 99-106.

Walton, D.M., Macdermid, J.C., Giorgianni, A.A., Mascarenhas, J.C., West, S.C. \& Zammit, C.A. (2013) Risk Factors for Persistent Problems Following Acute Whiplash Injury: Update of a Systematic Review and Meta-Analysis. J Orthop Sports Phys Ther.

Verhagen, A.P., Scholten-Peeters, G.G., van Wijngaarden, S., de Bie, R.A. \& Bierma-Zeinstra, S.M. (2007) Conservative treatments for whiplash. Cochrane Database Syst Rev, CD003338. 
Vernon, H. (2008) The Neck Disability Index: state-of-the-art, 1991-2008. J Manipulative Physiol Ther, 31, 491-502.

\section{Web references:}

Landén Ludvigsson M , Peolsson A., Peterson G (2015). DOI: http://dx.doi.org/10.3384/report.diva113865. Accessed 2015-03-15 
Eligibility, Assessment 1 (n=7950) Letters, including basic inclusion/exclusion criteria ${ }^{*}$, NDI and VAS, inviting participants were sent to individuals aged 18-63, identified from health care records in 6 counties in the preceding 6-36 months. Two reminders were sent.

Eligibility, Assessment 2 (n=419) A telephone interview was undertaken to confirm inclusion/exclusion criteria, and evaluate if further review of medical files was required to determine eligibility. Eligible participants then attended a physical examination to confirm WAD-grading ( 2 or 3 ).

Not eligible $(\mathbf{n}=7531)$

Reasons: did not meet self- reported inclusion criteria ${ }^{*}(\mathrm{n}=2173)$, VAS $<20 \mathrm{~mm} / \mathrm{NDI}<10$ ( $\left.\mathrm{n}=207\right)$, did not respond $(n=4548)$, addressee unknown $(n=314)$, fulfilled eligibility but declined to participate $(n=289)$

Excluded $(\mathbf{n}=203)$

Reasons: decline to participate due to lack of time $(n=37)$, other $(n=12)$, traumatic brain injury $(n=3)$ no whiplash injury $(n=15)$, other severe illness/main pain location elsewhere $(n=42),>3$ years since trauma $(n=37)$, previous unresolved neck injury/sick leave $>1$ months before trauma $(n=11)$, fracture/luxation/op $(n=4)$ insufficient knowledge of Swedish language $(n=16)$, relocated residence $(n=8)$, failed to attend physical examination/unable to contact $(n=18)$

\section{Group A}

Neck-specific exercise (NSE)

Allocated to intervention $(n=76)$

Never started intervention $(\mathrm{n}=6)$

\section{$\downarrow$}

Follow-up 3 months

Lost to follow-up $(n=12)$

Reasons: Lack of time/personal reasons $(n=4)$, increased pain $(n=1)$, other disease $(n=3)$ unknown $(n=4)$

Follow-up 12 months

Lost to follow-up $(n=18)$

Reasons: Lack of time/personal reasons $(n=5)$, increased pain $(n=1)$, other disease $(n=3)$ unknown $(n=9)$

Randomized (n=216)

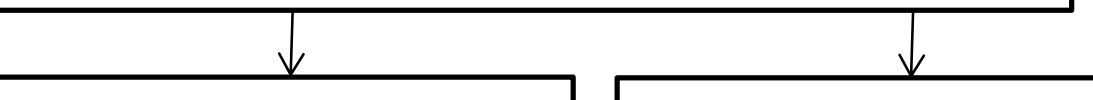

Group B

Neck-specific exercise with behavioral intervention (NSEB)

Allocated to intervention $(n=71)$

Never started intervention $(\mathrm{n}=3)$

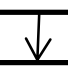

Follow-up 3 months

Lost to follow-up $(n=5)$

Reasons: Lack of time/personal reasons $(n=3)$, unknown $(n=1)$, moved $(\mathrm{n}=1)$

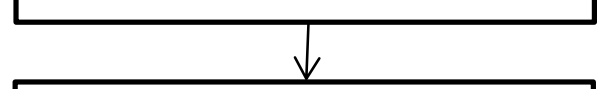

Follow-up 12 months

Lost to follow-up $(n=11)$

Reasons: Lack of time/personal reasons $(n=5)$, increased pain $(n=1)$, unknown $(n=3)$, moved $(n=2)$

\section{Group C}

Prescribed physical activity (PPA) Allocated to intervention $(n=69)$

Never started intervention $(n=5)$

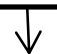

Follow-up 3 months

Lost to follow-up $(n=11)$

Reasons: Lack of time/personal reasons $(n=4)$, other disease $(n=4)$, unknown $(\mathrm{n}=3)$

\section{Follow-up 12 months}

Lost to follow-up $(n=17)$

Reasons: Lack of time/personal reasons $(n=5)$, unknown $(n=5)$, other disease $(n=5)$, moved $(n=2)$

\section{Figure 1.}


Figure legends

\section{Figure 1}

Participant flow through study

NSE $=$ Neck-specific exercise group, NSEB $=$ Neck-specific exercise with a behavioral approach group, PPA = Prescription of physical activity group. 
* whiplash injury in the preceding 6-36 months, reported to be the onset of current symptoms, excluding unconsciousness/loss of memory in connection to the whiplash injury, previous neck trauma with unresolved symptoms, previous neck surgery, ongoing malignant disease, severe psychiatric disorders, drug abuse, difficulties understanding the Swedish language. 